\section{On the degree of regularity of surfaces formed by the asymptotic integrals of differential equations}

\author{
by Z. SzmydT (Kraków)*
}

Consider the system of differential equations

$$
d \eta_{i} / d t=F^{(i)}\left(\eta_{1}, \ldots, \eta_{n}\right) \quad(i=1,2, \ldots, n)
$$

and assume that the origin of the coordinate system $\Theta_{n}=(0, \ldots, 0)$ is its singular point, i.e. that $F^{(i)}(0, \ldots, 0)=0 \quad(i=1,2, \ldots, n)$. Further, let us assume that all the characteristic roots of the matrix

$$
O=\left(c_{i j}\right), \quad e_{i j}=F_{\eta_{j}}^{(i)}(0, \ldots, 0) \quad(i, j=1,2, \ldots, n)
$$

have the real parts different from zero and that there exists at least one pair of roots whose real parts are of opposite signs. Let us denote by $S$ the set made up by the trajectories of the system $(0.1)$ tending to the singular point $\Theta_{n}$ for $t \rightarrow \infty$.

I. G. Petrovskil showed in 1934 [6], under fairly general assumptions regarding the regularity of functions $F^{(i)}(i=1,2, \ldots, n)$, that the set $S$ is a continuous manifold. In 1940, M. Martin proved the analyticity of the surface $S$, assuming that the functions $F^{(i)}(i=1,2, \ldots, n)$ are analytic and putting certain additional restrictions on the matrix $C$.

In the present paper we prove that the manifold $S$ is a surface of class $\sigma^{p}$ if the functions $F^{(i)}(i=1,2, \ldots, n)$ are of class $C^{p}(p \geqslant 1)$ (cf. theorem 1 , $\S 1$. Besides, we give an effective construction of the sequence of functions uniformly convergent to the function whose graph is the surface $S$. The derivatives up to the $p$ th order of the functions of the above sequence are uniformly convergent to the corresponding derivatives of the limit function.

The proof of theorem 1 is given in $\S 3$ (theorem 1 bis) in a special coordinate system (coordinates $z_{1}, z_{2}, \ldots, z_{n}$ ). The passage from the coordinates $\eta_{1}, \ldots, \eta_{n}$ to $z_{1}, \ldots, z_{n}$ is given in $\S 2$.

*) This result was communicated to the VIII Congress of Polish Mathemati. cians (September, 1953). It is also mentioned in the paper [7].
81. Definition 1. We say that the real matrix $C=\left(C_{i j}\right)(i, j=$ $=1,2, \ldots, n)$ is of type $T_{q}(0<q<n)$ if the numbers of the characteristic roots whose real parts are negative or positive are respectively $q$ and $n-q$.

Consider the system of differential equations

$$
d \eta_{i} \mid d t=\sum_{j=1}^{n} c_{i j} \eta_{j} \quad(i=1,2, \ldots, n)
$$

which, by setting

$$
H=\left(\eta_{1}, \ldots, \eta_{n}\right), \quad C=\left(c_{i j}\right)
$$

we may also write in the vectorial form

$$
d H / d t=C H .
$$

It is known (cf. [5], chapter III, p. 207) that if the matrix $C$ is of type $T_{q}$, the set made up by the trajectories of the system (1.2) tending to $\Theta_{n}=(0, \ldots, 0)$ for $t \rightarrow 0$ is a certain $q$-dimensional hyperplane passing through the origin of the coordinate system $\Theta_{n}$. We shall call it the negative characteristic hyperplane and denote it by $N$. Analogously we define the positive characteristic hyperplane $P$ as hyperplane of the trajectories of the system (1.2) tending to $\Theta_{n}$ for $t \rightarrow-\infty$.

Let a system of differential equations

$$
d \eta_{i} / d t=F^{(i)}\left(\eta_{1}, \ldots, \eta_{n}\right) \quad(i=1,2, \ldots, n)
$$

be given. By introducing the vector-function $F(H)=\left(F^{(1)}(H), \ldots, F^{(n)}(H)\right)$ (cf. (1.1)) we write the system (1.3) in the form

$$
d H / d t=F(H) .
$$

THeOREM 1. Let us assume that the function $H^{\prime}(H)$ is of class $C^{p}(p \geqslant 1)$ in the neighbourhood of $\Theta, F^{\prime}\left(\Theta_{n}\right)=0(i=1,2, \ldots, n)$, and that the matrix $C=\left(c_{i j}\right), c_{i j}=F_{n_{i}}^{(i)}\left(\Theta_{n}\right)(i, j=1,2, \ldots, n)$ is of the type $T_{q}$.

Under these hypotheses there exists a neighbourhood of the point $\Theta_{n}$ (a homeomorph of the n-dimensional open sphere) such that all the trajectories of the system (1.4) contained in this neighbourhood and tending to $\Theta_{\text {n }}$ for $t \rightarrow \infty$ make up a surface $S$ of class $C^{p}$ slight ${ }^{1}$ ) with respect to the hyperplane $N$ and tangent to it at $\Theta_{n}$ (interior point of $S$ ).

Remark 1 (cf. [7], remarque 4). By the transformation $\tau=-t$ we obtain from theorem 1 an analogous theorem for the set $R$ made up by the trajectories of the system (1.4) tending to $\Theta_{n}$ for $t \rightarrow-\infty$. From

1) This means that every hyperplane parallel to the positive characteristic hyperplane cuts the surface $S$ at most at one point. 
that follows the existence of a transformation of class $C^{p} \quad(p \geqslant 1)$ which transforms the system (1.4) into a new system in such a manner that the surfaces $S$ and $R$ are transformed into plane surfaces, $S^{*}$ and $R^{*}$, lying in the plane $\xi_{q+1}=\ldots=\xi_{n}=0$ and in the plane $\xi_{1}=\ldots=\xi_{q}=0$ respectively. This transformation enables us to reduce the investigation of the asymptotic behaviour of the integrals tending to $\Theta_{n}$ to the investigation of a system of the form

$$
d \xi_{i} / d t=G^{(i)}\left(\xi_{1}, \ldots, \xi_{q}, 0, \ldots, 0\right) \quad(i=1,2, \ldots, q)
$$

which has a knot at the point $\Theta_{n}$.

Remark 2. M. Martin [4] has shown the analycity of the manifolds $S$ and $R$ under the conditions of analycity of the function $F(H)$ and under a certain additional assumptions ${ }^{2}$ ) regarding the matrix $C$. From our theorem it follows, without these additional assumptions regarding the matrix $C$, that the surfaces $S$ and $R$ are of class $C^{\infty}$.

§ 2. Definition 2. Let $D=\left(d_{i j}\right) \quad(i, j=1,2, \ldots, n)$ be an arbitrary real matrix and $W=\left(w_{1}, \ldots, w_{n}\right)$ an arbitrary real vector. In the present paper by the symbols $\|D\|$ and $\|W\|$ we shall denote the square root of the sum of the squares of all elements, $i$. $e$.

$$
\|D\|=\left(\sum_{i=1}^{n} \sum_{j=1}^{n} d_{i j}^{2}\right)^{1 / 2}, \quad\|W\|=\left(\sum_{i=1}^{n} w_{i}^{2}\right)^{1 / 2} .
$$

In the proofs of the theorems we shall make use of the following inequalities, which are immediately implied by the above definition:

$$
\begin{gathered}
\left\|D_{1}+D_{2}\right\| \leqslant\left\|D_{1}\right\|+\left\|D_{2}\right\|, \quad\left\|D_{1} \cdot D_{2}\right\| \leqslant\left\|D_{1}\right\| \cdot\left\|D_{2}\right\|, \\
\left\|\int_{t_{0}}^{t} W(\tau) d \tau\right\| \leqslant \int_{t_{0}}^{t}\|W(\tau)\| d \tau \quad \text { for } \quad t \geqslant t_{0}
\end{gathered}
$$

under the hypothesis that $\int_{t_{0}}^{t}\|W(\tau)\| d \tau$ exists.

Let $Z=\left(z_{1}, \ldots, z_{n}\right)$ be a vector in the $n$-dimensional space and $q$ a positive integer $0<q<n$. Let us write

$$
Z=(X, Y), \quad X=\left(z_{1}, \ldots, z_{q}\right), \quad Y=\left(z_{\alpha+1}, \ldots, z_{n}\right) .
$$

We denote by $\Theta_{n}, \Theta_{q}, \Theta_{n-q}$ the origins of the coordinate systems $Z, X$ and $Y$ respectively.

Consider the system of differential equations written in the vectorial form

$$
d X / d t=A X+L^{(1)}(Z), \quad d Y / d t=B Y+L^{(2)}(Z),
$$

2) That is besides the assumption that the matrix $O$ is ot type $T_{q}$. in which $A, B$ are constant real square matrices and $L^{(i)}(Z)(i=1,2)$ are vector-functions.

HYротнеsIs $K_{p}$. We say that the system (2.1) satisfies the hypothesis $K_{p}(p \geqslant 1)$ if the following four conditions are fulfilled:

$\left.\mathrm{W}_{1}\right) \quad L^{(1)}\left(\Theta_{n}\right)=\Theta_{q}, \quad L^{(2)}\left(\Theta_{n}\right)=\Theta_{n-q}$,

$\mathrm{W}_{2}$ ) there exists a number $\lambda>0$ such that

$$
\left\|e^{\Delta t}\right\| \leqslant q e^{-\lambda t}, \quad\left\|e^{-B t}\right\| \leqslant(n-q) e^{-\lambda t} \quad \text { for } t \geqslant 0,
$$

$\left.\mathrm{W}_{3}\right) \quad U A U \leqslant-\lambda\|U\|^{2}$ for every vector $U=\left(u_{1}, \ldots, u_{q}\right)$,

$\left.\mathrm{W}_{4}\right)$ there exist constant matrices $G^{(i)}(i=1,2)$ and vector-functions $L^{(i)}(Z)(i=1,2)$ of class $C^{p}$ in the neighbourhood of $\Theta_{n}$ such that

$$
\begin{gathered}
L^{(1)}(Z)=G^{(1)} X+E^{(1)}(Z), \quad L^{(2)}(Z)=G^{(2)} Y+E^{(2)}(Z), \\
\left\|G^{(i)}\right\| \leqslant 4^{-1} \lambda n^{-3 / 2} \quad(i=1,2), \\
L_{z j}^{(1)}\left(\Theta_{n}\right)=\Theta_{q}, \quad E_{z_{j}}^{(2)}\left(\Theta_{n}\right)=\Theta_{n-q} \quad(j=1,2, \ldots, n) .
\end{gathered}
$$

Remark 3. From the condition $\mathrm{W}_{4}$ ) of hypothesis $K_{p}$ follows the existence of a positive number $R$ such that in the closed sphere with centre at $\Theta_{n}$ and radius $R$ the functions $I^{(i)}(Z)$ are of class $C^{p}$ and satisfy Lipschitz's condition

$$
\left\|L^{(i)}(Z)-L^{(i)}(\hat{Z})\right\| \leqslant 2^{-1} \lambda n^{-3 / 2}\|Z-\hat{Z}\| \quad(i=1,2) .
$$

Indeed since $G^{(1)}, G^{(2)}$ are constant matrices and the functions $\mathcal{L}^{(i)}(Z)(i=1,2)$ are of class $C^{p}$ in the neighbourhood of $\Theta_{n}$, it follows from $(2.2)$ that $L^{(i)}(Z)$ are of class $C^{p}$ in the neighbourhood of $\Theta_{n}$. Inequalities (2.5) are obtained from the relations (2.2)-(2.4) on the basis of the mean value theorem applied to the functions $\mathcal{E}^{(i)}(Z)(i=1,2)$; which are of class $C^{1}$ in the neighbourhood of $\Theta_{n}(p \geqslant 1)$.

Definition 3. We shall denote by $F_{R}$ the common part of the $n$-dimensional open sphere $\mathrm{V}\left(\Theta_{n}, R\right)$ with centre at $\Theta_{n}$ and radius $R$ and a cylinder defined by the inequality $\|X\|<R / 2 q$.

Definition 4 . We shall denote by $S$ the set formed by the trajectories of the system (2.1) which are contained in $F_{R}$ and tend to $\Theta_{n}$ for $t \rightarrow \infty$.

In $\S 3$ we shall prove the following theorem:

THEOREM 1 bis. If the system (2.1) satisfies the hypothesis $K_{p}$, then the set $S$ is a surface of class $C^{p}(p \geqslant 1)$ slight with respect to the hyperplane $Y=\Theta_{n-q}$ and tangent to it at the point $\Theta_{n}$ (interior point of $S$ ). 
To reduce the proof of theorem 1 to the proof of theorem 1 bis we make use of the following lemma:

LEMMA 1. If the functions $F^{(i)}(H)=F^{(i)}\left(\eta_{1}, \ldots, \eta_{n}\right) \quad(i=1,2, \ldots, n)$ are of class $C^{p}(p \geqslant 1)$ in the neighbourhood of $\Theta_{n}=(0, \ldots, 0), F^{(i)}\left(\Theta_{n}\right)=0$ $(i=1,2, \ldots, n)$ and the matrix $O=\left(c_{i j}\right), c_{i j}=F_{\eta_{j}}^{(i)}\left(\Theta_{n}\right)(i, j=1,2, \ldots, n)$ is of type $T_{q}$, then there exists a real non-singular constant matrix $M$, such that the linear transformation $Z=M H$ transforms the system (1.4) into the system (2.1), which satisfies the hypothesis $K_{p}$.

The proof of lemma 1, which is in fact based only on the classical theorem of Jordan on reducing a matrix to the canonical form, will be given in $\S 4$ at the end of the paper.

From lemma 1 it follows that the system (1.4), satisfying the assumptions of theorem 1, may be transformed by means of a suitable non-singular transformation $Z=M H$ with constant real coefficients into the system (2.1), which satisfies the hypothesis $K_{p}$. Since the negative characteristic hyperplane of the system

$$
d X / d t=\left[A+G^{(1)}\right] X, \quad d Y / d t=\left[B+G^{(2)}\right] Y,
$$

which satisfies the inequalities $\mathrm{W}_{2}$ ) and (2.3) is the hyperplane $Y=\Theta_{n-q}$, therefore, in view of the properties of the transformation $Z=M H$, it is evident that theorem 1 bis implies theorem 1 .

\$3. The proof of theorem 1 bis, to which this paragraph is devoted, will be preceded by some lemmas.

LEMIA 2. Suppose that the inequalities $\mathrm{W}_{2}$ ) with respect to the system of differential equations

$$
d X / d t=A X+L^{(1)}(Z), \quad d Y / d t=B Y+L^{(2)}(Z)
$$

are satisfied and the functions $L^{(i)}(Z)(i=1,2)$ are continuous for $\|Z\| \leqslant R$. Then each integral $Z(t)=(X(t), Y(t))$ of the system (3.1) such that

(3.2) $\quad\|Z(t)\| \leqslant R \quad$ for $t \geqslant t_{0}, \quad X\left(t_{0}\right)=\Xi, \quad \Xi=\left(\xi_{1}, \ldots, \xi_{q}\right)$,

satisfies the system of integral equations

$$
\begin{aligned}
& X(t)=\exp \left[A\left(t-t_{0}\right)\right] \Xi+\int_{t_{0}}^{t} \exp [A(t-\tau)] L^{(1)}[Z(\tau)] d \tau \\
& Y(t)=-\int_{t}^{\infty} \exp [-B(\tau-t)] L^{(2)}[Z(\tau)] d \tau .
\end{aligned}
$$

Proof. Every integral of the system (3.1) satisfying the second of the conditions (3.2) satisfies, for suitably chosen $V_{0}$, the system of integral equations

$$
\begin{gathered}
X(t)=\exp \left[A\left(t-t_{0}\right)\right] \Xi+\int_{t_{0}}^{t} \exp [A(t-\tau)] L^{(1)}[Z(\tau)] d \tau, \\
Y(t)=\exp \left[B\left(t-t_{0}\right)\right] V(t), \quad \text { where } \\
V(t)=V_{0}+\int_{t_{0}}^{t} \exp \left[-B\left(\tau-t_{0}\right)\right] L^{(2)}[Z(\tau)] d \tau .
\end{gathered}
$$

It follows that $Z(t)$ satisfies the first. of the equations (3.3). To show that the second is also satisfied let us observe that by $\|V(t)\|$ $\leqslant\left\|\exp \left[-B\left(t-t_{0}\right)\right]\right\| \cdot\|Y(t)\|$, inequality $\left.\mathrm{W}_{2}\right)$ and the first of the conditions (3.2) we obtain the inequality $\|V(t)\| \leqslant R(n-q) e^{-\lambda\left(t-t_{0}\right)}$, and hence $V(t) \rightarrow \Theta_{n-q}$ for $t \rightarrow \infty$. It follows that

$$
V_{0}=-\int_{t_{0}}^{\infty} \exp \left[-B\left(\tau-t_{0}\right)\right] L^{(2)}[Z(\tau)] d \tau
$$

which proves, together with (3.4), that $Z(t)$ satisfies also the second of the equations (3.3).

LEMMA 3. Suppose that the system (3.1) satisfies the hypothesis $K_{p}$. Let $R$ be a positive number such that the inequatities (2.5) (cf. remark 3) hold in the closed sphere with centre at $\Theta_{n}$ and radius $R$. Let us denote by $\omega$ the $q+1$ dimensional set of points $(t, \Xi)=\left(t, \xi_{1}, \ldots, \xi_{q}\right)$ defined by the inequalities
( $\omega)$$$
\|\Xi\|<r, \quad t_{0} \leqslant t<\infty \quad(r=R / 2 q) .
$$

We assert that there exists a function $Z(t, \Xi)=(X(t, \Xi), Y(t, \Xi))$ which has the following properties $\mathrm{P}_{1}$ ) and $\mathrm{P}_{2}$ ):

$\left.\mathrm{P}_{1}\right) Z(t, \Xi)$ is in the set $\omega$ the limit of uniformly convergent sequence of successive approximations ${ }^{\nu} Z(t, \Xi)=\left({ }^{\nu} X(t, \Xi),{ }^{p} Y(t, \Xi)\right)$ given by the relations

$$
{ }^{n+1} X(t, \Xi)=\exp \left[A\left(t-t_{0}\right)\right] \Xi+\int_{t_{0}}^{t} \exp [A(t-\tau)] L^{(1)}\left[{ }^{\nu} Z(\tau, \Xi)\right] d \tau,
$$

$$
{ }^{p+1} Y(t, \Xi)=-\int_{t}^{\infty} \exp [-B(\tau-t)] L^{(2)}\left[{ }^{v} Z(\tau, \Xi)\right] d \tau .
$$

For every fixed $\Xi(\|\Xi\|<r), Z(t, \Xi)$ is an integral of the system (3.1) and satisfies the conditions
(3.7) $\|Z(t, \Xi)\| \leqslant R \quad$ for $t \geqslant t_{0}, \quad Z(t, \Xi) \rightarrow \Theta_{n}$ for $\quad t \rightarrow \infty, \quad X\left(t_{0}, \Xi\right)=\Xi$.
If $\Xi=\Theta_{q}$ then

$$
Z\left(t, \Theta_{q}\right)=\Theta_{n} \quad \text { for } \quad t \geqslant t_{0}
$$


$\mathrm{P}_{2}$ ) For every fixed $t, t \geqslant t_{0}$ the function $Z(t, \Xi)$ is of the class $C^{\prime \prime}$ with respect to $\Xi=\left(\xi_{1}, \ldots, \xi_{q}\right)$ for $\|\boldsymbol{\Xi}\|<r$, where all the derivatives of order $1,2, \ldots, p$ of $Z(t, \Xi)$ are limits of uniformly converging sequences of the corresponding derivatives of $" Z(t, \Xi)$.

Proof. For the sake of clarity, we divide the proof of lemma 3 into four stages.

I. We assert that the functions of the sequence " $Z(t, \Xi)=\left({ }^{n} X(t, \Xi)\right.$, $\left.{ }^{\prime} Y(t, \Xi)\right)$ formally determined by the relations (3.5) and (3.6), are defined and continuous in the set $\omega$ for $1 \leqslant \nu<\infty$ and have the following properties $(\nu=1,2, \ldots)$ :

$$
\text { (3.9) } \quad\left\|^{\nu+1} Z(t, \Xi)-{ }^{\nu} Z(t, \Xi)\right\| \leqslant q r 2^{-\nu} \quad \text { in the set } \omega \text {, }
$$

(3.10) $\left\|^{p} Z(t, \Xi)\right\| \leqslant\left[1+2^{-1}+\ldots+2^{-(y-1)}\right] q^{r}<R$ in the set (l),

(3.11) $d\left({ }^{p+1} X\right) / d t=A^{{ }^{v+1}} X+L^{(1)}\left({ }^{v} Z\right), \quad d\left({ }^{p+1} Y\right) / d t=B{ }^{{ }^{p+1}} Y+L^{(2)}\left({ }^{p} Z\right)$,

$$
\begin{gathered}
{ }^{v} X\left(t_{0}, \Xi\right)=\Xi, \\
{ }^{v} Z(t, \Xi) \rightarrow \Theta_{n} \quad \text { for } t \rightarrow \infty, \\
{ }^{v} Z\left(t, \Theta_{q}\right)=\Theta_{n} \quad \text { for } t \geqslant t_{0} .
\end{gathered}
$$

Remark 4. From the assumptions $\mathrm{W}_{2}$ ) of hypothesis $K_{p}$ it follows at once that the relations (3.6) define the function ${ }^{p+1} Z(t, \Xi)$, which is continuous in the set $\omega$ provided ${ }^{\nu} Z(t, E)$ is a continuous function such that $\left\|^{\prime \prime} Z(t, \Xi)\right\| \leqslant R$ for $(t, \Xi) \in \omega$.

We make use of remark 4 in proving that the sequence of functions formally defined by (3.5) and (3.6) is infinite.

By (3.5) and inequality $\mathrm{W}_{2}$ ) we obtain, in view of $r=R / 2 q$, $\|\boldsymbol{Z}(t, \boldsymbol{\Xi})\|=\left\|^{\mathrm{I}} X\right\| \leqslant q \exp \left[-\lambda\left(t-t_{0}\right)\right]\|\boldsymbol{\Xi}\| \leqslant q r<R$ for $t \geqslant t_{0}$.

Thus the relations $(3.10),(3.13),(3.14)$ hold for $y=1$ and by remark 4 the relations (3.6) define the function ${ }^{2} Z(t, \Xi)=\left({ }^{2} X(t, \Xi),{ }^{2} Y(t, \Xi)\right)$, whence, by assumptions $\left.W_{2}\right), W_{1}$ ) and (2.5) (cf. remark 3 ), we obtain

$$
\begin{aligned}
\|\|^{2} Z-{ }^{1} Z \| \leqslant & \left\|^{2} X-{ }^{1} X\right\|+\left\|^{2} Y-{ }^{1} Y\right\| \\
\leqslant & q \int_{t_{0}}^{t} \exp [-\lambda(t-\tau)]\left\|L^{(1)}\left[{ }^{1} Z(\tau, \Xi)\right]\right\| d \tau+ \\
& +(n-q) \int_{t}^{\infty} \exp [-\lambda(\tau-t)]\left\|L^{(2)}\left[{ }^{1} Z(\tau, \Xi)\right]\right\| d \tau \\
\leqslant & \frac{q}{\lambda} \cdot \frac{\lambda}{2 n} q r+\frac{n-q}{\lambda} \cdot \frac{\lambda}{2 n} q r=\frac{q r}{2},
\end{aligned}
$$

which proves that (3.9) holds for $\nu=1$.

Let

We have

(3.16)

$$
{ }^{p+1} m=\sup _{t_{0} \leqslant t<\infty}\left\|^{p+1} Z(t, \Xi)-{ }^{n} Z(t, \Xi)\right\|
$$

$$
{ }^{\nu+1} m \leqslant{ }^{v} m / 2 \quad(\nu=1,2, \ldots)
$$

under the assumption that ${ }^{\nu-1} Z,{ }^{\nu} Z$ are continuous and that $\left\|^{\nu-1} Z\right\| \leqslant R$, $\left\|{ }^{\mu} Z\right\| \leqslant R$. Indeed, from (3.6) under the assumption that $\left\|^{\prime-1} Z\right\| \leqslant R$, $\left\|^{\prime} Z\right\| \leqslant R$ we obtain, by assumption (2.5) and remark 4 , the inequalities

$$
\left\|^{p+1} X-{ }^{\nu} X\right\| \leqslant q \int_{t_{0}}^{t} \exp [-\lambda(t-\tau)](\lambda / 2 n)\left\|^{\nu} Z-{ }^{\nu-1} Z\right\| d \tau \leqslant \leqslant^{\nu} m q / 2 n,
$$$$
\left\|^{p+1} Y-{ }^{\nu} Y\right\| \leqslant(n-q) \int_{t}^{\infty} \exp [-\lambda(\tau-t)](\lambda / 2 n)\left\|^{p} Z-{ }^{\nu-1} Z\right\| d \tau \leqslant{ }^{v} m(n-q) / 2 n
$$

from which it follows that $\left\|^{p+1} Z-{ }^{\nu} Z\right\| \leqslant^{v} m / 2$ for $t \geqslant t_{0}$. From this and by the definition (3.15) we immediately obtain the relation (3.16), which was to be shown.

Suppose that the functions ${ }^{n} Z(t, \Xi)(\nu=1,2, \ldots, p)$ are continuous in the set $\omega$ and the inequalities (3.9), (3.10), (3.14) hold for $v=1,2, \ldots, p-1$. We shall show that the relations (3.6) define the functions ${ }^{p+1} X,{ }^{p+1} Y$ and that the inequalities (3.9), (3.10), (3.14) hold for $\nu=p$. Indeed,

$$
\begin{aligned}
& \left\|{ }^{p} Z\right\| \leqslant\left\|^{p} Z-{ }^{p-1} Z\right\|+\left\|^{p-1} Z\right\| \\
& \quad \leqslant 2^{-(p-1)} q r+\left[1+2^{-1}+\ldots+2^{-(p-2)}\right] q r=\left[1+2^{-1}+\ldots+2^{-(p-1)}\right] q r
\end{aligned}
$$

It follows by $r=R / 2 q$ (cf. the definition of the set $\omega$ ) that $\left\|^{p} Z\right\| \leqslant R$, and thus, by remark 4 , the formulas (3.6) determine the functions ${ }^{p+1} X,{ }^{p+1} Y$, and since by the induction hypothesis we have also $\left\|^{p-1} Z\right\| \leqslant R$, we obtain from inequality (3.16)

$$
{ }^{p+1} m \leqslant{ }^{p} m / 2 .
$$

Since (3.9) holds for $\nu=p-1$, we obtain from (3.17) and definition (3.15) the inequality

$$
\left\|{ }^{p+1} Z-{ }^{p} Z\right\| \leqslant 2^{-p} q r \text { for } t \geqslant t_{0},
$$

and from (3.6), the induction hypothesis (3.14) and $W_{1}$ ) it follows that relation (3.14) holds also for $v=p$.

Thus we have proved the existence of the sequence ${ }^{n} Z(t, \Xi)=(" X(t, \Xi)$, $\left.{ }^{\nu} Y(t, \Xi)\right)$ satisfying the relations $(3.5),(3.6),(3.9),(3.10),(3.14)$ and the relations (3.11), (3.12), which are simple consequences of the relations (3.5) and (3.6). For the inductive proof of relation (3.13), which, as has been shown, is true for $\nu=1$, let us suppose that

$$
{ }^{p-1} Z(t, \Xi) \rightarrow \Theta_{n} \quad \text { for } \quad t \rightarrow \infty \text {. }
$$


Since $\left\|^{p-1} Z(t, \Xi)\right\| \leqslant R$, we obtain from the assumptions $\left.\left.\mathrm{W}_{1}\right), \mathrm{W}_{2}\right)$ and (2.5) the inequalities

$$
\begin{aligned}
& \left\|^{p} X\right\| \leqslant q \exp \left[-\lambda\left(t-t_{0}\right)\right] r+q(\lambda / 2 n) \int_{t_{0}}^{t} \exp [-\lambda(t-\tau)]\left\|^{p-1} Z(\tau, \Xi)\right\| d \tau, \\
& \left\|^{p} Y\right\| \leqslant(n-q)(\lambda / 2 n) \int_{t}^{\infty} \exp [-\lambda(\tau-t)]\left\|^{p-1} Z(\tau, \Xi)\right\| d \tau
\end{aligned}
$$

from which, by $\lambda>0$ and assumption (3.18), follows

$$
\left\|^{p} Z\right\| \leqslant\left\|^{p} X\right\|+\left\|^{p} Y\right\| \rightarrow 0 \text { for } t \rightarrow \infty
$$

on the basis of l'Hospital's theorem applied to the integrals

$$
\begin{aligned}
& J_{1}=\int_{t_{0}}^{t} \exp [-\lambda(t-\tau)]\left\|^{p-1} Z(\tau, \Xi)\right\| d \tau=e^{-\lambda t} \int_{t_{0}}^{t} e^{\lambda \tau}\left\|^{p-1} Z(\tau, \Xi)\right\| d \tau, \\
& J_{2}=\int_{i}^{\infty} \exp [-\lambda(\tau-t)]\left\|^{p-1} Z(\tau, \Xi)\right\| d \tau=e^{\lambda t} \int_{i}^{\infty} e^{-\lambda \tau}\left\|^{p-1} Z(\tau, \Xi)\right\| d \tau .
\end{aligned}
$$

The sequence $\left\{{ }^{n} Z(t, \Xi)\right\}$, which we hare defined, satisfies also the relations (3.13).

Now we pass to the second stage of the proof of lemma 1.

II. We assert that there exists a vectorial function $Z(t, \Xi)=(X(t, \Xi)$, $Y(t, \Xi))$ which is continuous in the set $\omega$ and satisfies the following conditions:
(3.19)

$$
{ }^{v} Z(t, \Xi) \Rightarrow Z(t, \Xi) \text { for } \nu \rightarrow \infty \text { in the set } \omega,
$$
(3.20) $\quad X\left(t_{0}, \Xi\right)=\Xi$,
(3.21) $\quad\|Z(t, \Xi)\| \leqslant R \quad$ in the set $\omega$,
(3.22) $\quad Z\left(t, \Theta_{q}\right)=\Theta_{n}$ for $t \geqslant t_{0}$,
(3.23) $\quad Z(t, \Xi) \rightarrow \Theta_{n} \quad$ for every fixed $\Xi$ when $t \rightarrow \infty \quad(\|\Xi\|<r)$,
(3.24) $\quad d\left({ }^{v} X\right) / d t \Rightarrow d X / d t, \quad d\left({ }^{v} Y\right) / d t \Rightarrow d Y / d t \quad$ in the set $\omega$.

The existence and the continuity of the function $Z(t, \Xi)$ satisfying the relation (3.19) follows from the uniform convergency of the series $\left.{ }^{1} Z(t, \Xi)+\sum_{\nu=1}^{n}{ }^{\nu+1} Z(t, \Xi)-{ }^{p} Z(t, \Xi)\right]$ (cf. (3.9)). The relations (3.20)-(3.22) follow from (3.19) by (3.12), (3.10), and (3.14) respectively. To prove (3.23) let us choose an arbitrary $\delta>0$ and find $k$ and $T_{g}$ so large that (cf. (3.19), (3.13))

$$
\begin{aligned}
& \left\|Z(t, \Xi)-{ }^{k} Z(t, \Xi)\right\| \leqslant \delta / 2 \quad \text { in the set } \omega, \\
& \left\|^{k} Z(t, \Xi)\right\| \leqslant \delta / 2 \text { for } t \geqslant T_{\Xi} \quad \text { ( } \Xi \text { being fixed). }
\end{aligned}
$$

From the above inequalities it follows at once that

$$
\|Z(t, \Xi)\| \leqslant \delta \text { for } t \geqslant T_{\Xi},
$$

which shows that relation (3.23) holds true.

From $(3.11),(3.19)$ and the uniform continuity of the function $L^{(i)}(Z)$ follows the uniform convergency of the sequences $\left\{d\left({ }^{v} X\right) / d t\right\},\left\{d\left({ }^{\nu} Y\right) / d t\right\}$, from which by applying once more relation (3.19) we obtain the relations (3.24).

III. From (3.11), (3.19), (3.24) we obtain

$$
d X / d t=A X+L^{(1)}(Z), \quad d Y / d t=B Y+L^{(2)}(Z),
$$

which, together with the relations $(3.20),(3.21),(3.23)$, proves that for every fixed $\Xi(\|\Xi\|<r), Z(t, \Xi)=(X(t, \Xi), Y(t, \Xi))$ is an integral of the system (3.1) satisfying the conditions (3.7). It follows, on the basis of $I$ and II (cf. in particular (3.19), (3.22)) that the property $\mathrm{P}_{1}$ ) holds true.

IV. In the proof ${ }^{3}$ ) of the property $\mathrm{P}_{2}$ ) we make use of the following theorem of the theory functions of real variable (theorem A) giving only an outline of its proof.

THEOREM A. If $\Xi=\left(\xi_{1}, \xi_{2}, \ldots, \xi_{q}\right)$,

(3.25) $\quad{ }^{\mathrm{W}}(\boldsymbol{E}) \Rightarrow \mathrm{W}(\boldsymbol{\Xi})$ for $\nu \rightarrow \infty$ in the sphere $\|\boldsymbol{\Xi}\|<r$

and all the derivatives of function ${ }^{*} W(\Xi)(\nu=1,2, \ldots)$ up to the order $p$ with respect to the variables $\xi_{1}, \xi_{2}, \ldots, \xi_{q}$ are bounded in common and have a common module of continuity ${ }^{4}$ ) in the sphere $\|\boldsymbol{E}\|<r$, then the limit function $W(\Xi)$ is of class $C^{p}$ in the open sphere $\|\Xi\|<r$ and all the derivatives up to the order $p$ of function $W(\Xi)$ are the limits of uniformly convergent sequences of the corresponding derivatives of functions ${ }^{n} W(\Xi)$.

Proof. Denote by ${ }^{\nu} F(\Xi)$ the vector made up of all derivatives of the function "W( $\boldsymbol{\Xi})$ up to the order $p$. By Arzela's theorem (cf. [1], p. 132) and the assumptions of theorem $A$ it follows that from every sequence $\left\{{ }^{a_{\nu}} F(\Xi)\right\}$ chosen from the sequence $\left\{{ }^{v} F(\Xi)\right\}$ one can choose a sequence $\left\{{ }^{\beta \nu} F^{\prime}(\boldsymbol{\Xi})\right\}$ which is uniformly convergent in the sphere $\|\Xi\|<r$. Thanks to the choice of the sequence $\left\{\alpha_{\nu}\right\}$ being arbitrary one can easily show

a) This proof has been simplified thanks to a certain remark of A. Plis.

4) Let $f(\Xi)$ be a real valued function defined in the set $\Omega$. Let us set $\sigma(\delta, f)$ $\sup |f(\Xi)-f(\hat{\Xi})|$ for all $\Xi, \hat{\Xi}_{\epsilon} \Omega$ such that $\varrho(\Xi, \hat{\Xi})<\delta$. The function $\sigma(\delta, f)$ is called the module of continuity of the function $f(\Xi)$ if $\sigma(\delta) \rightarrow 0$ when $\delta \rightarrow 0$. We say that the functions $f$ belonging to a certain family $F$ are equi-continuous in the set $\Omega$, that the functions $f$ belonging to a common module of continuity, if there exists a function $\sigma(\delta)$ or that they have a common $\lim \sigma(\delta)=0$ for $\delta \rightarrow 0$ and $\sigma(\delta, f) \leqslant \sigma(\delta)$ for every function $f$ belonging to the family $F$. 
(c.. [3], v. II, p. 127) on the basis of relation (3.25) and the definition of the function ${ }^{n} F(\xi)$ that all the derivatives up to the order $p$ of the sequence of functions ${ }^{\nu} W(\Xi)$ are uniformly convergent to the corresponding derivatives of the limit-function $W(\Xi)$. Thus the function $W(\Xi)$ is (cf. the assumptions of theorem $\mathrm{A}$ ) of class $C^{p}$ in the sphere $\|\Xi\|<r$.

Definition 5. We shall denote by $D^{k} W(\boldsymbol{\Xi})$ an arbitrary (fixed) derivative of the order $k$ of the function $W(\Xi)$ with respect to the variables $\xi_{1}, \xi_{2}, \ldots, \xi_{q}$.

From theorem A, definition 5 and relation (3.19) it follows at once that in order to prove the property $\mathrm{P}_{2}$ ) it is sufficient to show that the sequence $\left\{{ }^{v} Z(t, \Xi)\right\}$ has the following property $\left.\mathrm{P}_{2}^{*}\right)$ :

PROPERTY $\left.\mathrm{P}_{2}^{*}\right)$. There exist finite constants $a_{1}, a_{2}, \ldots, a_{p}$ and increasing functions $\sigma_{1}(u), \sigma_{2}(u), \ldots, \sigma_{p}(u)$ such that for every $(t, \Xi) \in \omega,(t, \hat{\Xi}) \in(1)$ the following inequalities hold:

(3.26) $\quad\left\|D^{k} \cdot{ }^{\nu} Z(t, \Xi)\right\| \leqslant a_{k} \quad$ for $\quad 1 \leqslant \nu<\infty \quad(k=1,2, \ldots, p)$,

$$
\begin{gathered}
\left\|D^{k \cdot} \cdot{ }^{p} Z(t, \boldsymbol{\Xi})-D^{k \cdot} \cdot{ }^{p} Z(t, \hat{\boldsymbol{\Xi}})\right\| \leqslant \sigma_{k}(\|\Xi-\hat{\Xi}\|) \quad \text { for } \quad \nu=1,2, \ldots, \\
\sigma_{k}(u) \rightarrow 0 \quad \text { for } u \rightarrow 0 \quad(k=1,2, \ldots, p) .
\end{gathered}
$$

Proof. First we show by induction that for every $(t, \Xi) \in \omega,(t, \hat{\Xi}) \in \omega$ we have

(3.28) $\quad\left\|^{p} Z(t, \Xi)-{ }^{\nu} Z(t, \hat{\Xi})\right\| \leqslant 2 q\|\Xi-\hat{\Xi}\| \quad(\nu=1,2, \ldots)$.

Indeed, from (3.5) and inequality $\mathrm{W}_{2}$ ) we obtain

$$
\left\|{ }^{1} Z(t, \Xi)-{ }^{1} Z(t, \hat{\Xi})\right\| \leqslant q\|\Xi-\hat{\Xi}\|,
$$

while (3.6) and (2.5) and inequality $\mathrm{W}_{2}$ ) imply the inequalities

$$
\begin{gathered}
\left\|^{k+1} X(t, \Xi)-{ }^{k+1} X(t, \hat{\Xi})\right\| \\
\leqslant q\|\Xi-\hat{\Xi}\|+q \int_{t_{0}}^{t} \exp [-\lambda(t-\tau)](\lambda / 2 n)\left\|^{k} Z(\tau, \Xi)-{ }^{k} Z(\tau, \hat{\Xi})\right\| d \tau, \\
\left\|^{k+1} Y(t, \Xi)-{ }^{k+1} Y(t, \hat{\Xi})\right\| \\
\leqslant(n-q) \int_{t}^{\infty} \exp [-\lambda(\tau-t)](\lambda / 2 n)\left\|^{k} Z(\tau, \Xi)-{ }^{k} Z(\tau, \hat{\Xi})\right\| d \tau,
\end{gathered}
$$

from which, under the assumption that (3.28) holds for $\nu=k$, we obtain the inequality

$$
\begin{aligned}
\left\|^{k+1} Z(t, \Xi)-{ }^{k+1} Z(t, \hat{\Xi})\right\| \leqslant & q\|\boldsymbol{\Xi}-\hat{\Xi}\|+(q / \lambda)(\lambda / 2 n) 2 q\|\boldsymbol{\Xi}-\hat{\Xi}\|+ \\
& +[(n-q) / \lambda](\lambda / 2 n) 2 q\|\boldsymbol{\Xi}-\hat{\Xi}\|=2 q\|\Xi-\hat{\Xi}\|
\end{aligned}
$$

which shows that (3.28) holds for $\nu=k+1$.
We shall denote $\left.{ }^{5}\right)$ by $L_{(Z)}^{(i)}(Z)$ the jacobian matrix of the vectorfunction $L^{(i)}(Z) \quad(i=1,2)$ with respect to the variables $Z=\left(z_{1}, \ldots, z_{n}\right)$. From assumption (2.5) we obtain

$$
\left\|L_{(Z)}^{(i)}(Z)\right\| \leqslant \lambda / 2 n \quad \text { for } \quad\|Z\| \leqslant R \quad(i=1,2)
$$

and from the uniform continuity of the function $L_{(Z)}^{(i)}(Z)$ in the bounded and closed set $\|Z\| \leqslant R$ follows the existence of an increasing function $\delta(r)$ such that

$$
\begin{aligned}
\left\|L_{(Z)}^{(i)}\left(Z_{1}\right)-L_{(Z)}^{(i)}\left(Z_{2}\right)\right\| \leqslant \delta\left(\left\|Z_{1}-Z_{2}\right\|\right), \\
\delta(r) \rightarrow 0 \quad \text { for } r \rightarrow 0 \quad(i=1,2) .
\end{aligned}
$$

From (3.28), (3.10), (3.30) and by the monotonicity of $\delta(r)$ we obtain the inequalities $(\nu=1,2, \ldots)$

(3.31) $\quad\left\|L_{(Z)}^{(i)}\left[{ }^{\nu} Z(t, \Xi)\right]-L_{(Z)}^{(i)}\left[{ }^{\nu} Z(t, \hat{\Xi})\right]\right\| \leqslant \delta(2 q\|\Xi-\hat{\Xi}\|) \quad(i=1,2)$,

from which, by $(3.29)$, it follows that for two vectors $W=\left(w_{1}, \ldots, w_{n}\right)$ and $\hat{W}=\left[\hat{w}_{1}, \ldots, \hat{w}_{n}\right)$ such that $\|W\| \leqslant \beta,\|\hat{W}\| \leqslant \beta$ the inequalities

$$
\begin{gathered}
\left.\|\left\{L_{(Z)}^{(i)}{ }^{\nu} Z(t, \Xi)\right]\right\} W-\left\{L_{(Z)}^{(i)}\left[{ }^{\nu} Z(t, \hat{\Xi})\right]\right\} \hat{W} \| \\
\leqslant(\lambda / 2 n)\|W-\hat{W}\|+\beta \delta(2 q\|\Xi-\hat{\Xi}\|) \quad(i=1,2 ; \quad \nu=1,2, \ldots)
\end{gathered}
$$

hold.

Now we shall prove the relations (3.26) and (3.27) for $k=1$, showing by induction for every $(t, \Xi) \epsilon \omega,(t, \hat{\Xi}) \epsilon \omega$ the inequalities

$$
\left\|D^{1} \cdot{ }^{v} Z(t, \Xi)\right\| \leqslant 2 q \quad(\nu=1,2, \ldots),
$$

(3.34) $\left\|D^{1} \cdot{ }^{\nu} Z(t, \Xi)-D^{1} \cdot{ }^{\nu} Z(t, \hat{\Xi})\right\| \leqslant 4 q n \lambda^{-1} \delta(2 q\|\boldsymbol{\Xi}-\hat{\boldsymbol{\Xi}}\|) \quad(v=1,2, \ldots)$.

The relations (3.33) and (3.34) hold for $\nu=1$ since from (3.5) and $\mathrm{W}_{2}$ ) we obtain

$$
\left\|D^{1} \cdot{ }^{1} Z(t, \Xi)\right\| \leqslant q, \quad\left\|D^{1} \cdot{ }^{1} Z(t, \Xi)-D^{1} \cdot{ }^{1} Z(t, \hat{\Xi})\right\|=0 .
$$

Assume that (3.33) and (3.34) hold for $\nu=1,2, \ldots, m$. To prove that they also hold for $\nu=m+1$, let us observe first that

(3.35) $\quad D^{1} \cdot\left\{L^{(i)}\left[{ }^{m} Z(\tau, \Xi)\right]\right\}=\left\{L_{(Z)}^{(i)}\left[{ }^{m} Z(\tau, \Xi)\right]\right\} D^{1} \cdot m Z(\tau, \Xi) \cdot \quad(i=1,2)$.

From (3.29) and the hypothesis of induction (3.33) it follows that

$$
\left\|\left\{L_{(Z)}^{(i)}\left[{ }^{m} Z(\tau, \Xi)\right]\right\} D^{1} \cdot{ }^{m} Z(\tau, \Xi)\right\| \leqslant 2 q \lambda / 2 n \quad(i=1,2)
$$

5) Since $p \geqslant 1$, the functions $L^{(i)}(Z)(i=1,2)$, which are by hypothesis of the class $C^{p}$ in the set $\|Z\| \leqslant R$, are of the class $C^{1}$ in this set. 
and by the hypothesis of induction (3.34) and inequality (3.32), in which we set $y=m, W=D^{1}{ }^{m} Z(t, \Xi), \hat{W}=D^{1} \cdot{ }^{m} Z(t, \hat{\Xi}), \beta=2 q$, we obtain

(3.37) $\left\|\left\{L_{(i)}^{(i)}\left[{ }^{m} Z(\tau, \Xi)\right]\right\} D^{1} \cdot{ }^{m} Z(\tau, \Xi)-\left\{L_{(Z)}^{(i)}\left[{ }^{m} Z(\tau, \hat{\Xi})\right]\right\} D^{1} \cdot{ }^{m} Z(\tau, \hat{\Xi})\right\|$

$\leqslant(\lambda / 2 n)(4 q n / \lambda) \delta(2 q\|\boldsymbol{\Xi}-\hat{\boldsymbol{\Xi}}\|)+2 q \delta(2 q\|\boldsymbol{\Xi}-\hat{\boldsymbol{\Xi}}\|)=4 q \delta(2 q\|\boldsymbol{\Xi}-\hat{\boldsymbol{\Xi}}\|)$.

From (3.6), (3.35)-(3.37) and $\mathrm{W}_{2}$ ) it follows (cf. [2], v. I, p. 233) that

$$
D^{1 \cdot m+1} X(t, \Xi)=\exp \left[A\left(t-t_{0}\right)\right] D^{1} \Xi+
$$

$$
+\int_{t_{0}}^{t} \exp [A(t-\tau)]\left\{L_{(Z)}^{(1)}\left[{ }^{m} Z(\tau, \Xi)\right]\right\} D^{1 \cdot m} Z(\tau, \Xi) d \tau,
$$

$$
D^{1 \cdot{ }^{m+1}} Y(t, \Xi)=-\int_{t}^{\infty} \exp [-B(\tau-t)]\left\{L_{(2)}^{(2)}\left[{ }^{m} Z(\tau, \Xi)\right]\right\} D^{1 \cdot m} Z(\tau, \Xi) d \tau
$$

and

$$
\begin{aligned}
& \left\|D^{1} \cdot{ }^{m+1} X(t, \Xi)\right\| \leqslant q+(q / \lambda) 2 q \lambda / 2 n, \\
& \left\|D^{1} \cdot{ }^{m+1} Y(t, \Xi)\right\| \leqslant[(n-q) / \lambda] 2 q \lambda / 2 n
\end{aligned}
$$

and

$$
\left\|D^{1} \cdot{ }^{m+1} X(t, \boldsymbol{\Xi})-D^{1} \cdot{ }^{m+1} X(t, \hat{\Xi})\right\| \leqslant(q / \lambda) 4 q \delta(2 q\|\boldsymbol{E}-\hat{\boldsymbol{\Xi}}\|),
$$

(3.40) $\left\|D^{1 . m+1} Z(t, \Xi)-D^{1 . m+1} \bar{X}(t, \hat{\Xi})\right\| \leqslant[(n-q) / \lambda] 4 q \delta(2 q\|\Xi-\hat{\Xi}\|)$.

From (3.39) we immediately obtain relation (3.33) for $\nu=m+1$ and from (3.40) relation (3.34) for $\nu=m+1$.

Assume that the relations (3.26) and (3.27), which are satisfied, as has been shown, for $k=1$, hold for $k=1,2, \ldots, h-1$, where $2 \leqslant h \leqslant p$. The proof of the property $\mathrm{P}_{2}^{*}$ ) will be finished if we show that they hold for $k=h$.

It is obvious that if the function " $Z(t, \Xi)$ has derivatives up to the order $h$ with respect to the variables $\xi_{1}, \ldots, \xi_{q}$, then

$$
D^{h} \cdot\left\{L^{(i)}\left[{ }^{\nu} Z(t, \Xi)\right]\right\}={ }^{\nu} F^{(i)}(t, \Xi)+\left\{L_{(i)}^{(i)}\left[{ }^{\nu} Z(t, \Xi)\right]\right\} D^{h \cdot \nu} Z(t, \Xi) \quad(i=1,2)
$$

where the functions ${ }^{\circ} F^{(i)}(t, \Xi) \quad(i=1,2)$ are aggregates of the product of derivatives up to order $h-1$ of the functions ${ }^{v} z_{j}(t, \xi)$ by the derivatives up to the order $h$ of functions $L^{(i)}(Z)$ in which ${ }^{\nu} Z(t, \Xi)$ is substituted for $Z$. These factors are, by our assumptions, bounded by constants independent of $\nu$ and have a common module of continuity independent of $\nu$. It follows that the functions ${ }^{\nu} F^{(i)}(t, \Xi)(i=1,2)$ are bounded by constants independent of

(3.42) $\quad\left\|T^{(i)}(t, \Xi)\right\| \leqslant M \quad$ in the set $\omega$ for $v=1,2, \ldots \quad(i=1,2)$ and the existence of an increasing function $\delta^{*}(r)$ such that

$$
\begin{gathered}
\left\|^{\nu} F^{(i)}(t, \Xi)-{ }^{v} F^{(i)}(t, \hat{\Xi})\right\| \leqslant \delta^{*}(\|\Xi-\hat{\Xi}\|), \\
1 \leqslant \nu<\infty \quad(i=1,2), \quad \delta^{*}(r) \rightarrow 0 \quad \text { for } \quad r \rightarrow 0 .
\end{gathered}
$$

We shall show by induction that for all positive integers $v$ the inequalities

$$
\begin{aligned}
& \left\|D^{h} \cdot{ }^{\nu} Z(t, \Xi)\right\| \leqslant 2 n M / \lambda, \\
& \left\|D^{h} \cdot{ }^{\nu} Z(t, \Xi)-D^{h} \cdot{ }^{\nu} Z(t, \hat{\Xi})\right\| \\
& \leqslant(2 n / \lambda)\left[\delta^{*}(\|\Xi-\hat{\Xi}\|)+(2 n M / \lambda) \delta(2 q\|\Xi-\hat{\Xi}\|)\right]
\end{aligned}
$$

hold, from which follow at once the relations (3.26) and (3.27) for $k=h$. For the proof we observe that from (3.5) we obtain the relations

$$
\left\|D^{h \cdot 1} Z(t, \Xi)\right\|=0, \quad\left\|D^{h}:{ }^{1} Z(t, \Xi)-D^{h} \cdot{ }^{1} Z(t, \hat{\Xi})\right\|=0
$$

which show that (3.44) and (3.45) hold for $y=1$. Assume that the inequalities (3.44) and (3.45) hold for every $\nu \leqslant m$ (the hypothesis of induction). From (3.29) and the hypothesis of induction (3.44) it follows that

$$
\begin{aligned}
& \left\|\left\{L_{(Z)}^{(i)}\left[{ }^{\nu} Z(t, \Xi)\right]\right\} D^{h} \cdot{ }^{\prime} Z(t, \Xi)\right\| \leqslant(\lambda / 2 n)(2 n M / \lambda)=M, \\
& v=1,2, \ldots, m, \quad i=1,2
\end{aligned}
$$

and by the hypothesis of induction (3.45) and the inequality (3.32), in which we set $W=D^{h \cdot v} Z(t, \Xi), \hat{W}=D^{h \cdot "} Z(t, \hat{\Xi}), \beta=2 n M / \lambda$, we obtain for $\nu=1,2, \ldots, m, i=1,2$

(3.47)

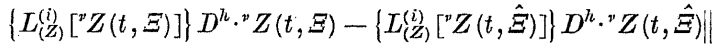

$$
\begin{aligned}
& \leqslant(\lambda / 2 n)(2 n / \hat{\lambda})\left[\delta^{*}(\|\boldsymbol{E}-\hat{\boldsymbol{\Xi}}\|)+(2 n \boldsymbol{M} / \lambda) \delta(2 q\|\boldsymbol{E}-\hat{\boldsymbol{\Xi}}\|)\right]+ \\
& +(2 n M / \lambda) \delta(2 q\|\Xi-\hat{\Xi}\|) \\
& =\delta^{*}(\|\Xi-\hat{\Xi}\|)+(4 n M / \lambda) \delta(2 q\|\Xi-\hat{\Xi}\|) .
\end{aligned}
$$

From (3.6), (3.41), (3.42), (3.46), (3.43), (3.47), it follows that (cf. [2], v. I, p. 233)

$$
\begin{aligned}
& D^{h \cdot{ }^{m+1}} X=\int_{t_{0}}^{t} \exp [A(t-\tau)]\left\{{ }^{(1} F^{(1)}(\tau, \Xi)+L_{(Z)}^{(1)}\left[{ }^{m} Z(\tau, \Xi)\right] D^{h \cdot{ }^{m}} Z(\tau, \Xi)\right\} d \tau, \\
& \text { (3.48) } \quad D^{h \cdot{ }^{m+1}} Y=-\int_{t}^{\infty} \exp [-B(\tau-t)]\left\{{ }^{p} F^{(2)}(\tau, \Xi)+\right. \\
& \left.+L_{(Z)}^{(2)}\left[{ }^{m} Z(\tau, \Xi)\right] D^{h \cdot m} Z(\tau, \Xi)\right\} d \tau \text {; }
\end{aligned}
$$


moreover, the inequalities

$$
\begin{gathered}
\left\|D^{h \cdot{ }^{m+1}} X\right\| \leqslant 2 q M / \lambda, \quad\left\|D^{h \cdot{ }^{m+1}} Y\right\| \leqslant 2(n-q) M / \lambda, \\
\left\|D^{h \cdot{ }^{m+1}} X(t, \Xi)-D^{h \cdot{ }^{m+1}} X(t, \hat{\Xi})\right\| \\
\leqslant(q / \lambda)\left\{2 \delta^{*}(\|\Xi-\hat{\Xi}\|)+(4 n M / \lambda) \delta(2 q\|\Xi-\hat{\Xi}\|)\right\} \\
\left\|D^{h \cdot{ }^{m+1}} Y(t, \Xi)-D^{h \cdot{ }^{m+1}} Y(t, \hat{\Xi})\right\| \\
\leqslant[(n-q) / \lambda]\left\{2 \delta^{*}(\|\Xi-\hat{\Xi}\|)+(4 n M / \lambda) \delta(2 q\|\Xi-\hat{\Xi}\|)\right\}
\end{gathered}
$$

hold.

Since (3.44) and (3.45) for $\nu=m+1$ follow from (3.49) and (3.50) respectively, therefore the inductive proof of the relations (3.44) and (3.45), and thus also of the property $\mathrm{P}_{2}^{*}$ ), is completed.

LEMMA 4. The system (3.1), satisfying the hypothesis $K_{p}$, has precisely one integral $Z(t, \Xi)=(X(t, \Xi), Y(t, \Xi))$ satisfying the conditions (3.7). For every fixed $t\left(t \geqslant t_{0}\right)$ the function $Z(t, \Xi)$ is of class $O^{p}$ with respect to $\boldsymbol{E}=\left(\xi_{1}, \xi_{2}, \ldots, \xi_{q}\right)$ for $\|\boldsymbol{E}\|<r$.

Proof. The existence of the integral $Z(t, \Xi)$, which has the properties mentioned in lemma 4 , follows from lemma 3 . Denote by $Z^{*}(t, \Xi)=$ $=\left(X^{*}(t, \Xi), Y^{*}(t, \Xi)\right)$ any integral of the system (3.1) satisfying the conditions (3.7). From lemma 2 and inequalities $W_{2}$ ) and (2.5) we obtain

$$
\begin{gathered}
\left\|X(t, \Xi)-X^{*}(t, \Xi)\right\| \\
\leqslant q \int_{t_{0}}^{t} \exp [-\lambda(t-\tau)](\lambda / 2 n)\left\|Z(\tau, \Xi)-Z^{*}(\tau, \Xi)\right\| d \tau, \\
\left\|\bar{Y}(t, \Xi)-X^{*}(t, \Xi)\right\| \\
\leqslant(n-q) \int_{t}^{\infty} \exp [-\lambda(\tau-t)](\lambda / 2 n)\left\|Z(\tau, \Xi)-Z^{*}(\tau, \Xi)\right\| d \tau .
\end{gathered}
$$

Let us set $m=\sup _{t_{0} \leqslant \tau<\infty}\left\|Z(\tau, \Xi)-Z^{*}(\tau, \Xi)\right\|$. From (3.51) we obtain

$$
\left\|\boldsymbol{Z}(t, \boldsymbol{\Xi})-Z^{*}(t, \boldsymbol{\Xi})\right\| \leqslant(q / \lambda)(\lambda / 2 n) m+[(n-q) / \lambda](\lambda / 2 n) m=m / 2,
$$

whence, by the definition of $m$, it follows that

$$
Z(\tau, \Xi)=Z^{*}(\tau, \Xi) \text { for } t_{0} \leqslant \tau<\infty .
$$

This inequality implies the unicity of the integral of the system (3.1) satisfying the conditions (3.7). Thus the proof of lemma 4 is finished.

Proof of theorem 1 bis. Let $Z(t, \Xi)$ be an integral of the system (3.1) satisfying the conditions (3.7). By lemma 4 there exists precisely one such integral, and function $Z\left(t_{0}, \Xi\right)$ and therefore also the function $Y\left(t_{0}, \boldsymbol{\Xi}\right)$ are of class $\sigma^{p}$ for $\|\Xi\|<R / 2 q$.
It follows that the function $(3.52)$

$$
\Psi(X)=Y\left(t_{0}, X\right)
$$

of the independent variable $X$ is of class $C^{p}$ for $\|X\|<R / 2 q$. By $\mathrm{W}_{3}$ ) one can prove that $Z(t, E) \in F_{R}$ for $t \geqslant t_{0}$ (see def. 3). Since the right sides of the system (3.1) are independent of the time $t$, it follows from the definition of the function $\Psi(X)$ that $Y=\Psi(X),\|X\|<$ $<R / 2 q$ is the equation of the set $S$ (cf. definition $4, \S 2$ ). To prove theorem 1 , bis it is sufficient to show the contact of the surface $S$ with the hyperplane $Y=\Theta_{n-q}$ at the point $\Theta_{n}$. Since the surface $S$ is of class $C^{p}(p \geqslant 1)$, it must be shown that $\Psi_{-x_{i}}\left(\Theta_{q}\right)=\Theta_{n-q}$ for $i=1,2, \ldots, q$, or, by (3.52) that

$$
Y_{x_{i}}\left(t_{0}, \Theta_{q}\right)=\Theta_{n-q} \quad(i=1,2, \ldots, q) .
$$

Let us observe that from lemma 2 it follows that

(3.54) $\quad Y(t, \Xi)=-\int_{t}^{\infty} \exp [-B(\tau-t)] L^{(2)}[Z(\tau, \Xi)] d \tau$.

Making use of (2.2), (2.4), (3.8) it is easy to show that

$$
\left\{\frac{\partial}{\partial \xi_{i}} L^{(2)}[Z(t, \Xi)]\right\}_{z=\Theta_{q}}=\left\{L_{(Z)}^{(2)}\left[Z\left(t, \Theta_{q}\right)\right]\right\} Z_{\xi_{i}}\left(t, \Theta_{q}\right)=G^{(2)} Y_{\xi_{i}}\left(t, \Theta_{q}\right)
$$

By (3.55) we obtain from (3.54) (cf. (3.29), (3.21), (3.33), $\left.\left.P_{2}\right), \mathrm{W}_{2}\right)$ )

$$
Y_{\xi_{i}}\left(t, \Theta_{q}\right)=-\int_{i}^{\infty} \exp [-B(\tau-t)] G^{(2)} Y_{\xi_{i}}\left(\tau, \Theta_{q}\right) d \tau
$$

From (3.56), $\left.\mathrm{W}_{2}\right),(2.3)$ it follows that

(3.57) $\left\|Y_{\xi_{i}}\left(t, \Theta_{q}\right)\right\| \leqslant(n-q)(\lambda / 4 n) \int_{t}^{\infty} \exp [-\lambda(\tau-t)]\left\|Y_{\xi_{i}}\left(\tau, \Theta_{q}\right)\right\| d \tau$ for $t \geqslant t_{0}$.

Let us set $m_{i}=\sup _{t_{0} \leqslant \tau<\infty}\left\|Y_{\xi_{i}}\left(\tau, \Theta_{q}\right)\right\|$. Now we obtain from (3.57)

$$
\left\|Y_{\xi_{i}}\left(t, \Theta_{q}\right)\right\| \leqslant[(n-q) \lambda / 4 n]\left(m_{i} \mid \lambda\right)<m_{i} / 4 \text { for } t \geqslant t_{0},
$$

whence, by the definition of $m_{i}$, follow the relations

$$
Y_{\xi_{i}}\left(t, \Theta_{q}\right)=\Theta_{n-q} \quad \text { for } \quad t \geqslant t_{0} \quad(i=1,2, \ldots, q),
$$

and thus in particular also the relations (3.53), which were to be shown.

\$ 4. To complete our considerations it suffices to prove lemma 1 only (cf. $\S 2$ ). To begin with we shall recall some theorems of the theory of matrices and linear transformations. 
Let $C=\left(c_{i j}\right)(i, j=1,2, \ldots, n)$ be an arbitrary real square matrix and $\lambda_{1}, \lambda_{2}, \ldots, \lambda_{n}$ the full sequence of all its characteristic roots. We assume that there exists a positive integer $q$ such that $0<q<n$ and

$$
\operatorname{Re} \lambda_{\mathrm{I}} \leqslant \ldots \leqslant \operatorname{Re} \lambda_{q}<\operatorname{Re} \lambda_{q+1} \leqslant \ldots \leqslant \operatorname{Re} \lambda_{n} .
$$

It is known (cf. [8], p. 295-296) that for every $\varepsilon>0$ there exists a non-singular matrix $M$ such that the matrix

$$
J=M C M^{-1}=\left(\begin{array}{ll}
J_{1} & 0 \\
0 & J_{2}
\end{array}\right)
$$

has Jordan's canonical form such that

1) $\lambda_{1}, \lambda_{2}, \ldots, \lambda_{q}$ is the sequence of all characteristic roots of the matrix $J_{1}$,

2) $\lambda_{q+1}, \lambda_{q+2}, \ldots, \lambda_{n}$ is the sequence of all characteristic roots of the matrix $\mathcal{J}_{2}$,

3 ) the matrices $J_{1}$ and $J_{2}$ have the form $K$

$$
K=\left[\begin{array}{rrrrrrr}
N_{1} L_{1} & & & & & \\
& N_{2} & L_{2} & & & & \\
& & \ddots & & & & \\
& & & N_{r-1} L_{r-1} & & \\
& & & N_{r} & & \\
& & & & \alpha_{2 r+1} & \varepsilon_{2 r+1} & \\
& & & & \ddots & \\
& & & & & \alpha_{s-1} & \varepsilon_{s-1} \\
& & & & & \alpha_{s}
\end{array}\right] \text {, }
$$

where

$$
N_{j}=\left[\begin{array}{rr}
\alpha_{j} & -\beta_{j} \\
\beta_{j} & \alpha_{j}
\end{array}\right], \quad L_{j}=\left[\begin{array}{ll}
\varepsilon_{j} & 0 \\
0 & \varepsilon_{j}
\end{array}\right] \quad(j=1,2, \ldots, r), \quad\left|\varepsilon_{k}\right|<\varepsilon
$$

for every index $k, \alpha_{k}(k=1, \ldots, s)$ and $\beta_{k}(k=1, \ldots, r)$, denote respectively the real and the imaginary parts of the roots of the matrix $C, \beta_{k} \neq 0$ and the vacant places are occupied by zeros.

Denote by $N$ the matrix

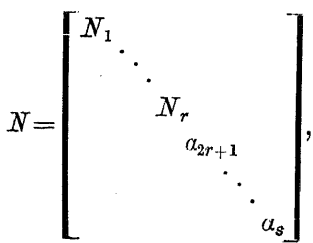

Obviously

$$
\|K-N\| \leqslant s^{1 / 2} \varepsilon
$$

and on account of the form of the matrix $e^{N t}$

$$
e^{N t}=\left[\begin{array}{ccc}
S_{1} & & \\
\ddots & & \\
& S_{r} & \\
& e^{\alpha_{2 r+1} t} \\
& & \ddots \\
& & e^{a_{s} t}
\end{array}\right], \quad \text { where } S_{j}=\left[\begin{array}{ll}
e^{a_{j} t} \cos \beta_{j} t-e^{a_{j} t} \sin \beta_{j} t \\
e^{a_{j} t} \sin \beta_{j} t & e^{a_{j} t} \cos \beta_{j} t
\end{array}\right]
$$

$(j=1,2, \ldots, r)$, the inequality

holds.

The above considerations lead to the following lemma:

LEMica 5. Let $C$ be a matrix of the type $T_{q}$ (cf. def. 1, $\$ 1$ ). Then there exists a number $\lambda>0$ (dependent on the matrix $C$ ) such that for every $\varepsilon>0$ one can find a real non-singular matrix $M$, and the $q$ matrices $A, G$, and $n-q$ matrices $B, H$, satisfying the following relations:

$$
\begin{gathered}
M C M^{-1}=\left[\begin{array}{ll}
J_{1} & 0 \\
0 & J_{2}
\end{array}\right] \quad J_{1}=A+G, \quad J_{2}=B+H, \\
\|G\| \leqslant q^{1 / 2} \varepsilon, \quad\|H\| \leqslant(n-q)^{1 / 2} \varepsilon, \\
\left\|e^{A t}\right\| \leqslant q e^{-\lambda t}, \quad\left\|e^{-B t}\right\| \leqslant(n-q) e^{-\lambda t} \quad \text { for } \quad t \geqslant 0, \\
U A U \leqslant-\lambda\|U\|^{2} \quad \text { for every vector } U=\left(u_{1}, \ldots, u_{q}\right) .
\end{gathered}
$$

Proof. Denote by $\lambda_{1}, \lambda_{2}, \ldots, \lambda_{n}$ the sequence of all characteristic roots of the matrix $C$ of type $T_{q}$ and assume that (cf. def. $1, \S 1$ )

$$
\operatorname{Re} \lambda_{1} \leqslant \operatorname{Re} \lambda_{2} \leqslant \ldots \leqslant \operatorname{Re} \lambda_{q}<0<\operatorname{Re} \lambda_{q+1} \leqslant \ldots \leqslant \operatorname{Re} \lambda_{n} .
$$

Let $\lambda$ be an arbitrary number satisfying the inequality

$$
0<\lambda<\left|\operatorname{Re} \lambda_{i}\right| \quad(i=1,2, \ldots, n) .
$$

The existence of matrices $A, B$ of type $N$ and of the matrices $G, H$, $M$ satisfying the relations (4.3) and (4.4) follows immediately from the considerations preceding lemma $5 . \lambda_{1}, \lambda_{2}, \ldots, \lambda_{q}$ and $\lambda_{q+1}, \lambda_{q+2}, \ldots, \lambda_{n}$ are sequences of all the characteristic roots of the matrices $A$ and $B$ res- 
pectively. The inequalities (4.5) hold by (4.6), (4.7), the inequalities of the form (4.2) and by the identity

$$
U N U=\alpha_{1}\left(u_{1}^{2}+u_{2}^{2}\right)+\ldots+\alpha_{r}\left(u_{2 r-1}^{2}+u_{2 r}^{2}\right)+\alpha_{2 r+1} a_{2 r+1}^{2}+\ldots+\alpha_{s} u_{s}^{2}
$$

satisfied by the matrices $A$ and $B$ of the type $N$.

Lemina 6. Assume that the matrix $C$ of the coefficients of the system of differential equations

$$
d H / d t=C H+\Phi(H)
$$

is of type $T_{q}$ and that $\Phi(H)$ is of class $C^{p}$ in the neighbourhood of $\Theta_{n}(p \geqslant 1)$

$$
\Phi\left(\Theta_{n}\right)=\Theta_{n},
$$

$$
\Phi_{\eta_{j}}\left(\Theta_{n}\right)=\Theta_{n} \quad(j=1,2, \ldots, n) .
$$

Then there exists a real non-singular linear transformation $Z=M H$, which transforms the system (4.8) into the system

$$
d X / d t=A X+L^{(1)}(Z), \quad d Y / d t=B Y+L^{(2)}(Z),
$$

where $Z=(X, Y)$, satisfying the hypothesis $K_{p}$.

Proof. Let $M$ be a matrix chosen according to lemma 5 for the matrix $C$ and the number $\varepsilon=\lambda / 4 n^{2}$. It follows that the linear transformation $Z=M H$ transforms the system (4.8) into the system (4.11), which satisfies the inequalities $W_{2}$ ) and the relations

$$
L^{(1)}(Z)=G^{(1)} X+L^{(1)}(Z), \quad L^{(2)}(Z)=G^{(2)} Y+E^{(2)}(Z)
$$

where $G^{(i)}$ are constant matrices satisfying the inequalities (2.3), and the function

$$
\text { (4.13) } \quad\left(L^{(1)}(Z), L^{(2)}(Z)\right)=M \Phi\left(M^{-1} Z\right)
$$

is of class $C^{p}$ in the neighbourhood of $\left.\Theta_{n}{ }^{6}\right)$. By (4.10) we obtain from the formula (4.13)

$$
\mathbb{L}_{z_{j}}^{(1)}\left(\Theta_{n}\right)=\Theta_{q} \quad E_{z_{j}}^{(2)}\left(\Theta_{n}\right)=\Theta_{n-q} \quad(j=1,2, \ldots, n)
$$

and since from the relations (4.12), (4.13), (4.9) it follows that $L^{(1)}\left(\Theta_{n}\right)=\Theta_{q}$, $L^{(2)}\left(\Theta_{n}\right)=\Phi_{n-q}$ therefore the proof of lemma 6 is complete.

Remark 5. Lemma 1 is the immediate consequence of lemma 6 . Indeed from the assumptions of lemma 1 it follows that the function $\Phi(H)$ defined by the relation $\Phi(H)=F(H)-C H, F(H)=\left(F^{(1)}(H), \ldots, F^{(n)}(H)\right)$ is of class $C^{p}$ in the neighbourhood of $\Theta_{n}$ and $\Phi\left(\Theta_{n}\right)=\Theta_{n}, \Phi_{\eta_{j}}\left(\Theta_{n}\right)=\Theta_{n}$ $(j=1,2, \ldots, n)$.

6) This follows from (4.13) since $\Phi(H)$ is of class $C^{p}$ in the neighbourhood of $\Theta_{n}$ and $M$ is a constant non-singular matrix.

\section{References}

[1] S. B anach, Wstep do teorii funkoji rzeczywistych, Warszawa-Wrocław 1951.

[2] E. Goursat, Cours d'analyse Mathématique, Paris 1923.

[3] O. Haupt, G. A umann, Differential- und Integralrechnung, Berlin 1938.

[4] M. Martin, Real asymptotic solutions of real differential equations, Bull. Amer. Math. Soc. 46 (1940), p. 475-481.

[5] В. В. Немыцки й и В. В. Степанов, Качественная теория дифференциальных уравнений, Москва 1949.

[6] I. G. Petrovskil, Über das Verhalten der Integralkurven eines Systems gewöhnlichen Differentialgleichungen in der Nähe eines singulären Punktes, Mat. Sbornik 41 (1934), 107-156.

[7] Z. Szmydt, Sur la structure de l'ensemble engendré par les intégrales tendant vers le point singulier du système d'équations différentielles, Bulletin de l'Académie Polonaise des Sciences, Cl. III, vol I, No 6 (1953), p. 223-227.

[8] T. Wà̇ewski, Sur les intégrales d'un système d'équations différentielles tangentes aux hyperplans caractéristiques issues du point singulier, Ann. Soc. Polon. Math. 21 (1948), p. 277-297.

\section{INSTYTUT MATEMATYCZNY POLSKIEJ AKADEMII NAUK}

MATHEMATICAL INSTITUTE OF THE POLISH ACADEMY OF SCIENCES 